\title{
EXISTENCE AND UNIQUENESS OF SOLUTIONS OF HIGHER-ORDER ANTIPERIODIC DYNAMIC EQUATIONS
}

\author{
ALBERTO CABADA AND DOLORES R. VIVERO \\ Received 8 October 2003 and in revised form 9 February 2004
}

We prove existence and uniqueness results in the presence of coupled lower and upper solutions for the general $n$th problem in time scales with linear dependence on the $i$ th $\Delta$ derivatives for $i=1,2, \ldots, n$, together with antiperiodic boundary value conditions. Here the nonlinear right-hand side of the equation is defined by a function $f(t, x)$ which is rd-continuous in $t$ and continuous in $x$ uniformly in $t$. To do that, we obtain the expression of the Green's function of a related linear operator in the space of the antiperiodic functions.

\section{Introduction}

The theory of dynamic equations has been introduced by Stefan Hilger in his Ph.D. thesis [12]. This new theory unifies difference and differential equations and has experienced an important growth in the last years. Recently, many papers devoted to the study of this kind of problems have been presented. In the monographs of Bohner and Peterson $[5,6]$ there are the fundamental tools to work with this type of equations. Surveys on this theory given by Agarwal et al. [2] and Agarwal et al. [1] give us an idea of the importance of this new field.

In this paper, we study the existence and uniqueness of solutions of the following $n$ thorder dynamic equation with antiperiodic boundary value conditions:

$\left(L_{n}\right)$

$$
\begin{gathered}
u^{\Delta^{n}}(t)+\sum_{j=1}^{n-1} M_{j} u^{\Delta^{j}}(t)=f(t, u(t)), \quad \forall t \in I=[a, b], \\
u^{\Delta^{i}}(a)=-u^{\Delta^{i}}(\sigma(b)), \quad 0 \leq i \leq n-1 .
\end{gathered}
$$

Here, $n \geq 1, M_{j} \in \mathbb{R}$ are given constants for $j \in\{1, \ldots, n-1\},[a, b]=\mathbb{T}^{\kappa^{n}}$, with $\mathbb{T} \subset \mathbb{R}$ an arbitrary bounded time scale and $f: I \times \mathbb{R} \rightarrow \mathbb{R}$ satisfies the following condition: 
(Hf) for all $x \in \mathbb{R}, f(\cdot, x) \in C_{\mathrm{rd}}(I)$ and $f(t, \cdot) \in C(\mathbb{R})$ uniformly at $t \in I$, that is, for all $\epsilon>0$, there exists $\delta>0$ such that

$$
|x-y|<\delta \Longrightarrow|f(t, x)-f(t, y)|<\epsilon, \quad \forall t \in I .
$$

A solution of problem $\left(L_{n}\right)$ will be a function $u: \mathbb{T} \rightarrow \mathbb{R}$ such that $u \in C_{\text {rd }}^{n}(I)$ and satisfies both equalities. Here, we denote by $C_{\mathrm{rd}}^{n}(I)$ the set of all functions $u: \mathbb{T} \rightarrow \mathbb{R}$ such that the $i$ th derivative is continuous in $\mathbb{T}^{\kappa^{i}}, i=0, \ldots, n-1$, and the $n$th derivative is rdcontinuous in $I$.

It is clear that for any given constant $M \in \mathbb{R}$, problem $\left(L_{n}\right)$ can be rewritten as

$$
\begin{gathered}
u^{\Delta^{n}}(t)+\sum_{j=1}^{n-1} M_{j} u^{\Delta^{j}}(t)+M u(t)=f(t, u(t))+M u(t), \quad \forall t \in I, \\
u^{\Delta^{i}}(a)=-u^{\Delta^{i}}(\sigma(b)), \quad 0 \leq i \leq n-1 .
\end{gathered}
$$

Defining the linear operator $T_{n}[M]: C_{\mathrm{rd}}^{n}(I) \rightarrow C_{\mathrm{rd}}(I)$ for every $u \in C_{\mathrm{rd}}^{n}(I)$ as

$$
T_{n}[M] u(t):=u^{\Delta^{n}}(t)+\sum_{j=1}^{n-1} M_{j} u^{\Delta^{j}}(t)+M u(t), \quad \text { for every } t \in I
$$

and the set

$$
W_{n}:=\left\{u \in C_{\mathrm{rd}}^{n}(I): u^{\Delta^{i}}(a)=-u^{\Delta^{i}}(\sigma(b)), 0 \leq i \leq n-1\right\}
$$

we can rewrite the dynamic equation $\left(L_{n}\right)$ as

$$
T_{n}[M] u(t)=f(t, u(t))+M u(t), \quad t \in I, u \in W_{n} .
$$

From this fact, we deduce that to ensure the existence and uniqueness of solutions of the dynamic equation $\left(L_{n}\right)$, we must determine the real values $M, M_{1}, \ldots, M_{n-1}$ for which the operator $T_{n}[M]$ is invertible on the set $W_{n}$, that is, the values for which Green's function associated with the operator $T_{n}^{-1}[M]$ in $W_{n}$ can be defined. In Section 2, we present the expression of Green's function associated to the operator $T^{-1}$ in $W_{n}$, where $T$ is a general $n$ th-order linear operator that is invertible on that set. This formula is analogous to the one given in [9] for $n$ th-order dynamic equations with periodic boundary value conditions.

In Section 3, we prove a sufficient condition for the existence and uniqueness of solutions of the dynamic equation $\left(L_{n}\right)$. For this, we take as reference the results obtained in $[3,4]$, where the existence and uniqueness of solutions of problem $\left(L_{n}\right)$ is studied in the particular case $\mathbb{T}=\{0,1, \ldots, P+n\}$ and so $\left(L_{n}\right)$ is a difference equation with antiperiodic boundary conditions. In this case, the classical iterative methods based on the existence of a lower and an upper solution and on comparison principles of some adequate linear 
operators, cannot be applied and, as a consequence, extremal solutions do not exist in a given function's set. Hence, to study the existence and uniqueness of solutions of problem $\left(L_{n}\right)$ in an arbitrary bounded time scale $\mathbb{T} \subset \mathbb{R}$, we use the technique developed in $[3,4]$, based on the concept of coupled lower and upper solutions, similar to the definition given in [10] for operators defined in abstract spaces and in [11] for antiperiodic boundary first-order differential equations. A survey of those results for difference equations can be founded in [8].

Using the results proved in Sections 2 and 3, we will obtain in Sections 4 and 5 the expression of Green's function and a sufficient condition for the existence and uniqueness of solutions of the dynamic equations of first- and second-order, respectively; likewise, we will give details about the continuous case where a dynamic equation is a differential equation and the discrete case, in which either a difference equation or a $q$-difference equation are treated.

\section{Expression of Green's function}

In this section, we obtain the expression of Green's function associated with the operator $T^{-1}$ in $W_{n}$, where $T$ is a general linear operator of $n$ th-order that is invertible on the mentioned set.

First, we introduce the concept of $n$ th-order regressive operator, see [5, Definition 5.89 and Theorem 5.91].

Definition 2.1. Let $M_{i} \in \mathbb{R}, 0 \leq i \leq n-1$ be given constants, the operator $T: C_{\mathrm{rd}}^{n}(I) \rightarrow$ $C_{\mathrm{rd}}(I)$, defined for every $u \in C_{\mathrm{rd}}^{n}(I)$ as

$$
T u(t):=u^{\Delta^{n}}(t)+\sum_{i=0}^{n-1} M_{i} u^{\Delta^{i}}(t), \quad \text { for every } t \in I,
$$

is regressive on $I$ if and only if $1+\sum_{i=1}^{n}(-\mu(t))^{i} M_{n-i} \neq 0$ for all $t \in I$.

Theorem 2.2. Let $M_{i} \in \mathbb{R}, 0 \leq i \leq n-1$ be given constants such that the operator $T$ defined in (2.1) is regressive on I (see Definition 2.1). If the operator $T$ is invertible on $W_{n}$, then Green's function associated to the operator $T^{-1}$ in $W_{n}, G: \mathbb{T} \times I \rightarrow \mathbb{R}$ is given by the following expression:

$$
G(t, s)= \begin{cases}u(t, s)+v(t, s), & \text { if } a \leq \sigma(s) \leq t \leq \sigma^{n}(b), \\ u(t, s), & \text { if } a \leq t<\sigma(s) \leq \sigma(b),\end{cases}
$$

where, for every $s \in[a, b]$ fixed, $v(\cdot, s)$ is the unique solution of the problem

$\left(Q_{s}\right)$

$$
\begin{gathered}
T x_{s}(t)=0, \quad t \in[\sigma(s), b], \\
x_{s}^{\Delta^{i}}(\sigma(s))=0, \quad i=0,1, \ldots, n-2, \\
x_{s}^{\Delta^{n-1}}(\sigma(s))=1,
\end{gathered}
$$

and for every $s \in[a, b]$ fixed, $u(\cdot, s)$ is given as the unique solution of the problem 
$\left(R_{s}\right)$

$$
\begin{gathered}
T y_{s}(t)=0, \quad t \in[a, b] \\
y_{s}^{\Delta^{i}}(a)+y_{s}^{\Delta^{i}}(\sigma(b))=-v^{\Delta^{i}}(\sigma(b), s), \quad i=0,1, \ldots, n-1 .
\end{gathered}
$$

Proof. First, we see that the function $G$ is well defined, that is, for every $s \in[a, b]$ fixed, problems $\left(Q_{s}\right)$ and $\left(R_{s}\right)$ have a unique solution.

Since the operator $T$ is regressive on $I$, we have, see [5, Corollary 5.90 and Theorem 5.91], that for every $s \in[a, b]$ fixed, the initial value problem $\left(Q_{s}\right)$ has a unique solution.

To verify that the periodic boundary problem $\left(R_{s}\right)$ is uniquely solvable, we consider the following boundary value problem:

$\left(P_{\lambda}\right)$

$$
\begin{gathered}
w^{\Delta^{n}}(t)+\sum_{i=0}^{n-1} M_{i} w^{\Delta^{i}}(t)=h(t), \quad t \in I, \\
w^{\Delta^{i}}(a)+w^{\Delta^{i}}(\sigma(b))=\lambda_{i}, \quad i=0,1, \ldots, n-1,
\end{gathered}
$$

with $h \in C_{\mathrm{rd}}(I)$ and $\lambda_{i} \in \mathbb{R}, 0 \leq i \leq n-1$ fixed.

We know that $w \in C_{\mathrm{rd}}^{n}(I)$ is a solution of problem $\left(P_{\lambda}\right)$ if and only if $W(t)=$ $\left(w(t), w^{\Delta}(t), \ldots, w^{\Delta^{n-1}}(t)\right)^{T}$ is a solution of the matrix equation

$$
W^{\Delta}(t)=A W(t)+H(t), \quad t \in I, \quad W(a)+W(\sigma(b))=\lambda,
$$

where $H(t)=(0, \ldots, 0, h(t))^{T}, \lambda=\left(\lambda_{0}, \ldots, \lambda_{n-1}\right)^{T}$, and

$$
A=\left(\begin{array}{ccccc}
0 & 1 & 0 & \cdots & 0 \\
0 & 0 & 1 & \cdots & 0 \\
\vdots & \vdots & \vdots & \ddots & \vdots \\
0 & 0 & 0 & \cdots & 1 \\
-M_{0} & -M_{1} & -M_{2} & \cdots & -M_{n-1}
\end{array}\right) .
$$

Since the operator $T$ is regressive on $I$, we have, by [5, Definitions 5.5 and 5.89], that the matrix $A$ is regressive on $I$ too and so, it follows from [5, Theorem 5.24] that the initial value problem

$$
W^{\Delta}(t)=A W(t)+H(t), \quad t \in I, \quad W(a)=W_{a},
$$

has a unique solution that is given by the following expression:

$$
W(t)=e_{A}(t, a) W_{a}+\int_{a}^{t} e_{A}(t, \sigma(s)) H(s) \Delta s .
$$


If we denote the $n \times n$ identity matrix by $I_{n}$, then we obtain, from the boundary conditions, that problem (2.6) has a unique solution if and only if there exists a unique $W_{a}=W(a) \in \mathbb{R}^{n}$ such that

$$
\left(I_{n}+e_{A}(\sigma(b), a)\right) W_{a}=\lambda-\int_{a}^{\sigma(b)} e_{A}(\sigma(b), \sigma(s)) H(s) \Delta s,
$$

or equivalently, if and only if the matrix $I_{n}+e_{A}(\sigma(b), a)$ is invertible.

Now, since the operator $T$ is invertible on $W_{n}$, we have that problem $\left(P_{0}\right)$ has a unique solution and then there exists the inverse of such matrix. As a consequence, problem $\left(R_{s}\right)$ has a unique solution.

Now, let $z: \mathbb{T} \rightarrow \mathbb{R}$ be defined for every $t \in \mathbb{T}$ as

$$
z(t)=\int_{a}^{\sigma(b)} G(t, s) h(s) \Delta s .
$$

It is not difficult to prove, by using [5, Theorem 1.117], that $z$ is the unique solution of the problem $\left(P_{0}\right)$.

Now, we prove the following properties of Green's function associated to the operator $T^{-1}$ in $W_{n}$.

Proposition 2.3. Let $M_{i} \in \mathbb{R}, 0 \leq i \leq n-1$ be given constants such that the operator $T$ defined in (2.1) is regressive on I. If $G: \mathbb{T} \times I \rightarrow \mathbb{R}$ is Green's function associated to the operator $T^{-1}$ in $W_{n}$, defined in (2.2), then the following conditions are satisfied.

(1) There exists $k>0$ such that $|G(t, s)| \leq k$ for all $(t, s) \in \mathbb{T} \times I$.

(2) If $n=1$, then for every $s \in I$, the function $G(\cdot, s)$ is continuous at $t \in \mathbb{T}$ except at $t=s=\sigma(s)$.

(3) If $n>1$, then for every $s \in I$, the function $G(\cdot, s)$ is continuous in $\mathbb{T}$.

(4) If $n=1$, then for every $t \in \mathbb{T}$, the function $G(t, \cdot)$ is $r d$-continuous at $s \in I$ except when $s=t=\sigma(t)$.

(5) If $n>1$, then for every $t \in \mathbb{T}$, the function $G(t, \cdot)$ is $r d$-continuous in $I$.

Proof. As we have seen in the proof of Theorem 2.2, we know that Green's function associated to the operator $T^{-1}$ in $W_{n}$ is given as the $1 \times n$ term of the matrix function

$$
F(t, s)= \begin{cases}e_{A}(t, \sigma(s))-e_{A}(t, a)\left(I_{n}+e_{A}(\sigma(b), a)\right)^{-1} e_{A}(\sigma(b), \sigma(s)), & \sigma(s) \leq t, \\ -e_{A}(t, a)\left(I_{n}+e_{A}(\sigma(b), a)\right)^{-1} e_{A}(\sigma(b), \sigma(s)), & t<\sigma(s),\end{cases}
$$

where $A$ is the matrix given in (2.7).

From [5, Definition 5.18 and Theorem 5.23], we know that the matrix exponential function is continuous in both variables and so the function $G$ is bounded in the compact set $\mathbb{T} \times I$.

Now, since $e_{A}(t, t)=I_{n}$, if $t=\sigma(s)=s$, then the diagonal terms of $F(\cdot, s)$ are not continuous at $t$.

It is clear that in any other situation, the function $F(\cdot, s)$ is continuous at $t$. 
On the other hand, given $t_{0} \in \mathbb{T}$, for every $s_{0} \in I$ such that $s_{0} \neq t_{0}$, it follows, from the continuity of the exponential function, that if $s \rightarrow s_{0}$ and $\sigma(s) \rightarrow \sigma\left(s_{0}\right)$, then $F\left(t_{0}, s\right) \rightarrow$ $F\left(t_{0}, s_{0}\right)$.

Hence, since $G(t, s)\left(\equiv F_{1, n}(t, s)\right)$ belongs to the diagonal of $F(t, s)$ only when $n=1$, the properties (2), (3), (4), and (5) of the statement hold.

\section{Existence and uniqueness results}

In this section, we prove existence and uniqueness results for the $n$ th-order nonlinear dynamic equation with antiperiodic boundary conditions $\left(L_{n}\right)$.

Suppose that the function $f: I \times \mathbb{R} \rightarrow \mathbb{R}$ satisfies condition $(H f)$, the operator $T_{n}[M]$ is regressive on $I$ and invertible on $W_{n}$ and $G$ is Green's function associated to the operator $T_{n}{ }^{-1}[M]$ in $W_{n}$, defined in (2.2).

We define the functions $G^{+}, G^{-}: \mathbb{T} \times I \rightarrow \mathbb{R}$ as

$$
G^{+}:=\max \{G, 0\} \geq 0, \quad G^{-}:=-\min \{G, 0\} \geq 0,
$$

and so,

$$
G=G^{+}-G^{-} \quad \text { on } \mathbb{T} \times I
$$

Considering the operators $A_{n}^{+}[M], A_{n}^{-}[M]: C(\mathbb{T}) \rightarrow C(\mathbb{T})$ defined for every $\eta \in C(\mathbb{T})$ as

$$
\begin{aligned}
& A_{n}^{+}[M] \eta(t):=\int_{a}^{\sigma(b)} G^{+}(t, s)(f(s, \eta(s))+M \eta(s)) \Delta s, \quad t \in \mathbb{T}, \\
& A_{n}^{-}[M] \eta(t):=\int_{a}^{\sigma(b)} G^{-}(t, s)(f(s, \eta(s))+M \eta(s)) \Delta s, \quad t \in \mathbb{T},
\end{aligned}
$$

the solutions of the dynamic equation $\left(L_{n}\right)$ are the fixed points of the operator

$$
A_{n}[M]:=A_{n}^{+}[M]-A_{n}^{-}[M]
$$

Note that if condition $(H f)$ holds, then the operators $A_{n}^{+}[M]$ and $A_{n}^{-}[M]$ are well defined.

To deduce the existence and uniqueness of solutions of the dynamic equation $\left(L_{n}\right)$, we introduce the concept of coupled lower and upper solutions for such problem.

Definition 3.1. Given $M \in \mathbb{R}$ such that the operator $T_{n}[M]$ is regressive on $I$ and invertible on $W_{n}$, a pair of functions $\alpha, \beta \in C_{\mathrm{rd}}^{n}(I)$ such that $\alpha \leq \beta$ in $\mathbb{T}$ is a pair of coupled lower and upper solutions of the dynamic equation $\left(L_{n}\right)$ if the inequalities

$$
\begin{array}{ll}
\alpha(t) \leq A_{n}^{+}[M] \alpha(t)-A_{n}^{-}[M] \beta(t), & \forall t \in \mathbb{T}, \\
\beta(t) \geq A_{n}^{+}[M] \beta(t)-A_{n}^{-}[M] \alpha(t), & \forall t \in \mathbb{T},
\end{array}
$$

hold. 
Under the conditions of the previous definition, if $\alpha$ and $\beta$ are a pair of coupled lower and upper solutions for the dynamic equation $\left(L_{n}\right)$, then defining the operator

$$
B[M]:[\alpha, \beta] \times[\alpha, \beta] \longrightarrow C(\mathbb{T})
$$

as

$$
B[M](\eta, \xi):=A_{n}^{+}[M] \eta-A_{n}^{-}[M] \xi,
$$

and considering the hypothesis

(H) for every $t \in I$ and $\alpha(t) \leq u \leq v \leq \beta(t)$, it is satisfied that

$$
f(t, u)+M u \leq f(t, v)+M v,
$$

we prove the following monotonicity property.

Lemma 3.2. Suppose that $M \in \mathbb{R}$ is a given constant such that the operator $T_{n}[M]$ is regressive on $I$ and invertible on $W_{n}, \alpha$ and $\beta$ are a pair of coupled lower and upper solutions of the dynamic equation $\left(L_{n}\right)$ and the function $f: I \times \mathbb{R} \rightarrow \mathbb{R}$ satisfies hypotheses $(H f)$ and $(H)$. Then, $B[M](\eta, \xi) \in[\alpha, \beta]$ for all $\eta, \xi \in[\alpha, \beta]$. Moreover, if $\alpha \leq \eta_{1} \leq \eta_{2} \leq \beta$ and $\alpha \leq \xi_{2} \leq \xi_{1} \leq \beta$, then

$$
B[M]\left(\eta_{1}, \xi_{1}\right) \leq B[M]\left(\eta_{2}, \xi_{2}\right) \quad \text { in } \mathbb{T} .
$$

Proof. Let $\alpha \leq \eta_{1} \leq \eta_{2} \leq \beta$ and $\alpha \leq \xi_{2} \leq \xi_{1} \leq \beta$. It follows, from the definitions of $A_{n}^{+}[M]$ and $A_{n}^{-}[M]$, that

$$
\begin{array}{ll}
A_{n}^{+}[M] \alpha \leq A_{n}^{+}[M] \eta_{1} \leq A_{n}^{+}[M] \eta_{2} \leq A_{n}^{+}[M] \beta & \text { in } \mathbb{T} \\
A_{n}^{-}[M] \alpha \leq A_{n}^{-}[M] \xi_{2} \leq A_{n}^{-}[M] \xi_{1} \leq A_{n}^{-}[M] \beta & \text { in } \mathbb{T} .
\end{array}
$$

From the definitions of $\alpha$ and $\beta$, we obtain that

$$
\begin{aligned}
\alpha & \leq A_{n}^{+}[M] \alpha-A_{n}^{-}[M] \beta \leq A_{n}^{+}[M] \eta_{1}-A_{n}^{-}[M] \xi_{1} \\
& \leq A_{n}^{+}[M] \eta_{2}-A_{n}^{-}[M] \xi_{2} \leq A_{n}^{+}[M] \beta-A_{n}^{-}[M] \alpha \leq \beta \quad \text { in } \mathbb{T} .
\end{aligned}
$$

This completes the proof.

Now, we obtain a result which gives us a region where all the solutions in $[\alpha, \beta]$ of the dynamic equation $\left(L_{n}\right)$ lie.

Proposition 3.3. Suppose that $M \in \mathbb{R}$ is a given constant such that the operator $T_{n}[M]$ is regressive on I and invertible on $W_{n}, \alpha$ and $\beta$ are a pair of coupled lower and upper solutions of the dynamic equation $\left(L_{n}\right)$ and the function $f: I \times \mathbb{R} \rightarrow \mathbb{R}$ satisfies hypotheses $(H f)$ and $(H)$.

Then, there exist two monotone sequences in $C(\mathbb{T}),\left\{\varphi_{m}\right\}_{m \in \mathbb{N}}$, and $\left\{\psi_{m}\right\}_{m \in \mathbb{N}}$, with $\alpha=$ $\varphi_{0} \leq \varphi_{m} \leq \psi_{l} \leq \psi_{0}=\beta$ in $\mathbb{T}, m, l \in \mathbb{N}$ which converge uniformly to the functions $\varphi$ and $\psi$ that satisfy

$$
\varphi=A_{n}^{+}[M] \varphi-A_{n}^{-}[M] \psi, \quad \psi=A_{n}^{+}[M] \psi-A_{n}^{-}[M] \varphi \quad \text { in } \mathbb{T} .
$$


Moreover, any solution $u \in[\alpha, \beta]$ of $\left(L_{n}\right)$ belongs to the sector $[\varphi, \psi]$. If, in addition, $\varphi=\psi$, then $\varphi$ is the unique solution of $\left(L_{n}\right)$ in $[\alpha, \beta]$.

Proof. The sequences $\left\{\varphi_{m}\right\}_{m \in \mathbb{N}}$ and $\left\{\psi_{m}\right\}_{m \in \mathbb{N}}$ are obtained recursively as $\varphi_{0}:=\alpha, \psi_{0}:=\beta$ and for every $m \geq 1$,

$$
\varphi_{m}:=B[M]\left(\varphi_{m-1}, \psi_{m-1}\right), \quad \psi_{m}:=B[M]\left(\psi_{m-1}, \varphi_{m-1}\right)
$$

From Lemma 3.2, we know that $\alpha=: \varphi_{0} \leq \varphi_{1} \leq \psi_{1} \leq \psi_{0}:=\beta$ in $\mathbb{T}$.

By induction, we conclude that the sequence $\left\{\varphi_{m}\right\}_{m \in \mathbb{N}}$ is monotone increasing, the sequence $\left\{\psi_{m}\right\}_{m \in \mathbb{N}}$ is monotone decreasing, and $\varphi_{m} \leq \psi_{l}$ in $\mathbb{T}$ for every $m, l \in \mathbb{N}$.

As a consequence, for every $t \in \mathbb{T}$, there exist $\varphi(t):=\lim _{m \rightarrow \infty} \varphi_{m}(t)$ and $\psi(t):=$ $\lim _{m \rightarrow \infty} \psi_{m}(t)$.

From hypothesis $(\mathrm{Hf})$ and Proposition 2.3, we know that both sequences are uniformly equicontinuous on $I$ and so, Ascoli-Arzelà's theorem, (see [7, page 72], [14, page $735]$ ), implies that such convergence is uniform in $\mathbb{T}$. Now, [13, Theorem 1.4.3] shows that

$$
\varphi=A_{n}^{+}[M] \varphi-A_{n}^{-}[M] \psi, \quad \psi=A_{n}^{+}[M] \psi-A_{n}^{-}[M] \varphi \quad \text { in } \mathbb{T} .
$$

Let $u$ be a solution of the dynamic equation $\left(L_{n}\right)$ such that $u \in[\alpha, \beta]$. From Lemma 3.2 , we know that

$$
\varphi_{1}:=B[M](\alpha, \beta) \leq B[M](u, u)=u \leq B[M](\beta, \alpha)=: \psi_{1} \quad \text { in } \mathbb{T} .
$$

By recurrence, we arrive at $\varphi_{m} \leq u \leq \psi_{l}$ in $\mathbb{T}$ for all $m, l \in \mathbb{N}$. Thus, passing to the limit, we obtain that $\varphi \leq u \leq \psi$ in $\mathbb{T}$.

Finally, if $\varphi=\psi$, then we have that $\varphi=A_{n}^{+}[M] \varphi-A_{n}^{-}[M] \varphi=: A_{n}[M] \varphi$, that is, $\varphi=\psi$ is a solution of the dynamic equation $\left(L_{n}\right)$ in $[\alpha, \beta]$. Since all solutions of $\left(L_{n}\right)$ that belong to $[\alpha, \beta]$ lie in the sector $[\varphi, \psi]$, we conclude that $\varphi$ is the unique solution of $\left(L_{n}\right)$ in $[\alpha, \beta]$.

Now, let $\|\cdot\|$ be the supremum norm in $C(\mathbb{T})$.

We prove the following existence result, that gives us a sufficient condition to assure that the dynamic equation $\left(L_{n}\right)$ has a unique solution lying between a pair of coupled lower and upper solutions of $\left(L_{n}\right)$.

Theorem 3.4. Assume that $M \in \mathbb{R}$ is a given constant such that the operator $T_{n}[M]$ is regressive on $I$ and invertible on $W_{n}, \alpha$ and $\beta$ are a pair of coupled lower and upper solutions of the dynamic equation $\left(L_{n}\right)$ and the function $f: I \times \mathbb{R} \rightarrow \mathbb{R}$ satisfies hypothesis $(H f)$.

If for every $t \in I$ and $\alpha(t) \leq u \leq v \leq \beta(t)$ the inequalities

$$
-M(v-u) \leq f(t, v)-f(t, u) \leq(K-M)(v-u)
$$


are satisfied for some $K \geq 0$ such that

$$
K \cdot\left\|\int_{a}^{\sigma(b)}|G(t, s)| \Delta s\right\|<1,
$$

then the dynamic equation $\left(L_{n}\right)$ has a unique solution in $[\alpha, \beta]$.

Proof. Since the first part of the inequality (3.16) is hypothesis $(H)$, we know, by Proposition 3.3, that there exists a pair of functions $\varphi, \psi \in C(\mathbb{T})$ such that for every $t \in \mathbb{T}$ we have

$$
\begin{aligned}
0 \leq & (\psi-\varphi)(t) \\
= & A_{n}^{+}[M] \psi(t)-A_{n}^{-}[M] \varphi(t)-A_{n}^{+}[M] \varphi(t)+A_{n}^{-}[M] \psi(t) \\
= & \int_{a}^{\sigma(b)} G^{+}(t, s)[f(s, \psi(s))-f(s, \varphi(s))+M(\psi(s)-\varphi(s))] \Delta s \\
& +\int_{a}^{\sigma(b)} G^{-}(t, s)[f(s, \psi(s))-f(s, \varphi(s))+M(\psi(s)-\varphi(s))] \Delta s \\
= & \int_{a}^{\sigma(b)}|G(t, s)|[f(s, \psi(s))-f(s, \varphi(s))+M(\psi(s)-\varphi(s))] \Delta s \\
\leq & \int_{a}^{\sigma(b)}|G(t, s)| \cdot K \cdot(\psi(s)-\varphi(s)) \Delta s \\
\leq & \|\psi-\varphi\| \cdot K \cdot \| \int_{a}^{\sigma(b)}|G(t, s)| \Delta s \mid .
\end{aligned}
$$

Thus, it follows from the inequality (3.17) that $\varphi=\psi$ in $\mathbb{T}$ and Proposition 3.3 allows us to conclude that the dynamic equation $\left(L_{n}\right)$ has a unique solution in $[\alpha, \beta]$.

Remark 3.5. One can check, following the proofs given in these sections, that we can develop an analogous theory for problem

$\left(\bar{L}_{n}\right)$

$$
\begin{gathered}
-u^{\Delta^{n}}(t)+\sum_{j=1}^{n-1} M_{j} u^{\Delta^{j}}(t)=f(t, u(t)), \quad \forall t \in I=[a, b], \\
u^{\Delta^{i}}(a)=-u^{\Delta^{i}}(\sigma(b)), \quad 0 \leq i \leq n-1 .
\end{gathered}
$$

In this case, we must study the operator

$$
\bar{T}_{n}[M] u \equiv-u^{\Delta^{n}}+\sum_{j=1}^{n-1} M_{j} u^{\Delta^{j}}+M u
$$

in the space $W_{n}$.

The functions $\alpha$ and $\beta$ are given as in Definition 3.1, with $G$ Green's function related with operator $\bar{T}_{n}[M]$ in $W_{n}$. 


\section{First-order equations}

In this section, using the previously obtained results, we give a sufficient condition to ensure the existence and uniqueness of solutions of the first-order nonlinear dynamic equation with antiperiodic boundary conditions

$\left(L_{1}\right)$

$$
\begin{gathered}
u^{\Delta}(t)=f(t, u(t)), \quad \forall t \in I=[a, b], \\
u(a)=-u(\sigma(b)),
\end{gathered}
$$

where $f: I \times \mathbb{R} \rightarrow \mathbb{R}$ is a function that satisfies hypothesis $(H f)$ and $[a, b]=\mathbb{T}^{\kappa}$, with $\mathbb{T} \subset \mathbb{R}$ an arbitrary bounded time scale.

As we have noted in the previous section, to deduce the existence and uniqueness of solutions of $\left(L_{1}\right)$, we must study Green's function related with the dynamic equation

$$
u^{\Delta}(t)+M u(t)=h(t), \quad \forall t \in I, \quad u(a)=-u(\sigma(b)),
$$

with $h \in C_{\text {rd }}(I)$.

As we have seen in the proof of Theorem 2.2, we know that if $1-M \mu(t) \neq 0$ for all $t \in I$ and $1+e_{-M}(\sigma(b), a) \neq 0$, then the operator

$$
T_{1}[M] u(t):=u^{\Delta}(t)+M u(t), \quad \forall t \in I,
$$

is regressive on $I$ and invertible on $W_{1}$ and the dynamic equation (4.2) has a unique solution $z: \mathbb{T} \rightarrow \mathbb{R}$, defined for every $t \in \mathbb{T}$ as

$$
z(t)=\int_{a}^{\sigma(b)} G(t, s) h(s) \Delta s .
$$

It is not difficult to verify that the function $G$ is given by the expression

$$
G(t, s)= \begin{cases}\frac{e_{-M}(t, \sigma(s))}{1+e_{-M}(\sigma(b), a)}, & \text { if } a \leq \sigma(s) \leq t \leq \sigma(b), \\ -\frac{e_{-M}(t, a) e_{-M}(\sigma(b), \sigma(s))}{1+e_{-M}(\sigma(b), a)}, & \text { if } a \leq t<\sigma(s) \leq \sigma(b) .\end{cases}
$$

From [5, Theorem 2.44], we know that if $1-M \mu(t)>0$ for all $t \in I$, then $e_{-M}(t, s)>0$ for all $(t, s) \in \mathbb{T} \times I$, so that we only consider such situation.

From the expression of $G$, we obtain the following equalities.

(i) If $M=0$, then we have that

$$
\left\|\int_{a}^{\sigma(b)}|G(t, s)| \Delta s\right\|=\frac{\sigma(b)-a}{2} .
$$

(ii) If $M \neq 0$, then we have that

$$
\left\|\int_{a}^{\sigma(b)}|G(t, s)| \Delta s\right\|=\frac{1-e_{-M}(\sigma(b), a)}{M\left(1+e_{-M}(\sigma(b), a)\right)} .
$$


Therefore, from Theorem 3.4 we obtain the following result that assures the existence and uniqueness of solutions of the dynamic equation $\left(L_{1}\right)$ in the sector $[\alpha, \beta]$, with $\alpha$ and $\beta$ a pair of coupled lower and upper solutions of $\left(L_{1}\right)$.

Corollary 4.1. Assume that $M \in \mathbb{R}$ is such that $M<1 / \mu(t)$ for all $t \in I, \alpha$ and $\beta$ are a pair of coupled lower and upper solutions of $\left(L_{1}\right)$, and the function $f: I \times \mathbb{R} \rightarrow \mathbb{R}$ satisfies hypothesis $(\mathrm{H} f)$. If property (3.16) holds for some $K \geq 0$ such that

$$
K<\frac{2}{\sigma(b)-a}, \quad \text { if } M=0,
$$

or

$$
K<\frac{M\left(1+e_{-M}(\sigma(b), a)\right)}{1-e_{-M}(\sigma(b), a)}, \quad \text { if } M \neq 0,
$$

then the dynamic equation $\left(L_{1}\right)$ has a unique solution in $[\alpha, \beta]$.

4.1. Particular cases. Here, we consider differential, difference, and $q$-difference equations as particular situations.

Differential equations. Let $T>0$ and $\mathbb{T}=[0, T] \subset \mathbb{R}$. In this case, given $u:[0, T] \rightarrow \mathbb{R}$, it follows from [5, Theorem 1.16] that $u$ is $\Delta$-differentiable at $t \in[0, T]$ if and only if $u$ is differentiable (in the classical sense) at $t$ and, moreover, $u^{\Delta}(t)=u^{\prime}(t)$.

Since for every $M \in \mathbb{R}$ fixed we have that

$$
e_{M}\left(t, t_{0}\right)=e^{M\left(t-t_{0}\right)}, \quad \forall t, t_{0} \in \mathbb{T},
$$

we know that

$$
G(t, s)= \begin{cases}\frac{e^{-M(t-s)}}{1+e^{-M T}}, & \text { if } 0 \leq s<t \leq T, \\ -\frac{e^{-M(T+t-s)}}{1+e^{-M T}}, & \text { if } 0 \leq t \leq s \leq T .\end{cases}
$$

Thus, taking into account that in this case condition $(\mathrm{Hf})$ is equivalent to the continuity of the function $f$ in $I \times \mathbb{R}$, from Corollary 4.1 we arrive at the following result.

Corollary 4.2. Assume that $M \in \mathbb{R}$ is a given constant, $\alpha$ and $\beta$ are a pair of coupled lower and upper solutions of the differential equation $\left(L_{1}\right)$, and the function $f: I \times \mathbb{R} \rightarrow \mathbb{R}$ is continuous. If condition (3.16) is satisfied for some $K \geq 0$ such that

$$
K<\frac{2}{T}, \quad \text { if } M=0,
$$

or

$$
K<\frac{M\left(1+e^{-M T}\right)}{1-e^{-M T}}, \quad \text { if } M \neq 0,
$$

then the differential equation $\left(L_{1}\right)$ has a unique solution in $[\alpha, \beta]$.

Difference equations. Let $h>0, P \in\{1,2, \ldots\}$ and $\mathbb{T}=\{0, h, \ldots, h P\} \subset \mathbb{R}$. 
Given $u: \mathbb{T} \rightarrow \mathbb{R}$, it follows from [5, Theorem 1.16] that for every $t \in \mathbb{T}^{\kappa}, u$ is $\Delta$ differentiable at $t$, moreover, it is satisfied that

$$
u^{\Delta}(t)=\frac{u(t+h)-u(t)}{h}
$$

and for every $M \in \mathbb{R}, M \neq-1 / h$ fixed, we have that

$$
e_{M}\left(t, t_{0}\right)=(1+M h)^{\left(t-t_{0}\right) / h}, \quad \forall t, t_{0} \in \mathbb{T}
$$

As a consequence, we have that for all $M \in \mathbb{R}$ such that $M \neq 1 / h$ and $1+(1-M h)^{P} \neq 0$, the operator

$$
T_{1}[M] u(t):=\frac{u(t+h)+(M h-1) u(t)}{h}, \quad t \in I,
$$

is regressive on $I$ and invertible on $W_{1}$.

So, Green's function is given by the expression

$$
G(t, s)= \begin{cases}\frac{(1-M h)^{(t-s-h) / h}}{1+(1-M h)^{P}}, & \text { if } 0 \leq s+h \leq t \leq h P, \\ -\frac{(1-M h)^{(P h+t-s-h) / h}}{1+(1-M h)^{P}}, & \text { if } 0 \leq t \leq s \leq h(P-1),\end{cases}
$$

and we deduce the following result.

Corollary 4.3. Let $M \in \mathbb{R}$ be such that $M<1 / h, \alpha$ and $\beta$ a pair of coupled lower and upper solutions of the dynamic equation $\left(L_{1}\right)$, and the function $f: I \times \mathbb{R} \rightarrow \mathbb{R}$ is such that for every $t \in I, f(t, \cdot) \in C(\mathbb{R})$. If condition (3.16) is fulfilled for some $K \geq 0$ such that

$$
K<\frac{2}{h P}, \quad \text { if } M=0,
$$

or

$$
K<\frac{M\left(1+(1-M h)^{P}\right)}{1-(1-M h)^{P}}, \quad \text { if } M \neq 0,
$$

then the dynamic equation $\left(L_{1}\right)$ has a unique solution in $[\alpha, \beta]$.

$q$-difference equations. Given $q \in \mathbb{R}, q>1$, and $N \in \mathbb{N}, N \geq 1$; let $\mathbb{T}=\left\{1, q, \ldots, q^{N}\right\} \subset \mathbb{R}$.

If $u: \mathbb{T} \rightarrow \mathbb{R}$, then we know by [5, Theorem 1.16] that for every $t \in \mathbb{T}^{\kappa}, u$ is $\Delta$-differentiable at $t$ and

$$
u^{\Delta}(t)=\frac{u(q t)-u(t)}{(q-1) t} .
$$


Fix $M \in \mathbb{R}$ such that $M \neq-1 /\left((q-1) q^{k}\right)$ for every $k \in J_{N-1}=\{0,1, \ldots, N-1\}$. If $t=q^{i}$ and $s=q^{j}$, then we obtain that the exponential function is given by

$$
e_{M}(t, s)= \begin{cases}\prod_{k=j}^{i-1}\left(1+M(q-1) q^{k}\right), & \text { if } 0 \leq j<i \leq N, \\ 1, & \text { if } 0 \leq j=i \leq N, \\ \prod_{k=i}^{j-1} \frac{1}{\left(1+M(q-1) q^{k}\right)}, & \text { if } 0 \leq i<j \leq N .\end{cases}
$$

Thus, for all $M \in \mathbb{R}$ such that $M \neq 1 /\left((q-1) q^{k}\right)$ for every $k \in J_{N-1}$ and $1+\prod_{k=0}^{N-1}(1-$ $\left.M(q-1) q^{k}\right) \neq 0$, we arrive at the following expression for Green's function

$$
G(t, s)= \begin{cases}\frac{\prod_{k=j+1}^{i-1}\left(1-M(q-1) q^{k}\right)}{1+\prod_{k=0}^{N-1}\left(1-M(q-1) q^{k}\right)}, & \text { if } 0 \leq j+1 \leq i \leq N, \\ -\frac{\prod_{k \in I_{i, j}}\left(1-M(q-1) q^{k}\right)}{1+\prod_{k=0}^{N-1}\left(1-M(q-1) q^{k}\right)}, & \text { if } 0 \leq i \leq j \leq N-1,\end{cases}
$$

where we denote

$$
I_{i, j}=\{0, \ldots, i-1\} \cup\{j+1, \ldots, N-1\} .
$$

We obtain, from Corollary 4.1, the following result.

Corollary 4.4. Suppose that $M \in \mathbb{R}$ is such that $M<1 /\left((q-1) q^{N-1}\right), \alpha$ and $\beta$ are a pair of coupled lower and upper solutions of $\left(L_{1}\right)$, and $f: I \times \mathbb{R} \rightarrow \mathbb{R}$ satisfies $f(t, \cdot) \in C(\mathbb{R})$, for every $t \in I$. If condition (3.16) is true for some $K \geq 0$ such that

$$
K<\frac{2}{q^{N}-1}, \quad \text { if } M=0,
$$

or

$$
K<\frac{M\left(1+\prod_{k=0}^{N-1}\left(1-M(q-1) q^{k}\right)\right)}{1-\prod_{k=0}^{N-1}\left(1-M(q-1) q^{k}\right)}, \quad \text { if } M \neq 0,
$$

then the dynamic equation $\left(L_{1}\right)$ has a unique solution in $[\alpha, \beta]$.

\section{Second-order equations}

In this section, by using Remark 3.5 we give a sufficient condition for the existence and uniqueness of solutions of the second-order nonlinear dynamic equation with antiperiodic boundary conditions 
$\left(\bar{L}_{2}\right)$

$$
\begin{aligned}
&-u^{\Delta^{2}}(t)= f(t, u(t)), \quad \forall t \in I=[a, b], \\
& u(a)=-u(\sigma(b)), \\
& u^{\Delta}(a)=-u^{\Delta}(\sigma(b)),
\end{aligned}
$$

where $f: I \times \mathbb{R} \rightarrow \mathbb{R}$ satisfies hypothesis $(H f)$ and $[a, b]=\mathbb{T}^{\kappa^{2}}$, with $\mathbb{T} \subset \mathbb{R}$ an arbitrary bounded time scale.

In this case, we study the existence and uniqueness of solutions of the second-order linear dynamic equation

$(\bar{P})$

$$
\begin{gathered}
-u^{\Delta^{2}}(t)+M^{2} u(t)=h(t), \quad \forall t \in I, \\
u(a)=-u(\sigma(b)), \\
u^{\Delta}(a)=-u^{\Delta}(\sigma(b)),
\end{gathered}
$$

with $h \in C_{\text {rd }}(I)$.

We know, by Theorem 2.2 and Remark 3.5, that if $1-M^{2} \mu^{2}(t) \neq 0$ for every $t \in I$ and the operator

$$
\bar{T}_{2}[M] u(t):=-u^{\Delta^{2}}(t)+M^{2} u(t), \quad t \in I,
$$

is invertible on $W_{2}$, then the dynamic equation $(\bar{P})$ has a unique solution given by expression (2.11).

It is not difficult to verify that if $M=0$, then the expression of Green's function is given by

$$
G(t, s)= \begin{cases}\frac{1}{2}\left[\frac{1}{2}(\sigma(b)-a)-t+\sigma(s)\right], & \text { if } a \leq \sigma(s) \leq t \leq \sigma^{2}(b), \\ \frac{1}{2}\left[\frac{1}{2}(\sigma(b)-a)-\sigma(s)+t\right], & \text { if } a \leq t<\sigma(s) \leq \sigma(b) .\end{cases}
$$

Using this expression, for every $t \in[a, \sigma(b)]$, we obtain the following upper bound:

$$
\int_{a}^{\sigma(b)}|G(t, s)| \Delta s \leq \frac{5(\sigma(b)-a)^{2}}{4}=: K_{0,1}
$$

and, if $t=\sigma^{2}(b)>\sigma(b)$, then we have that

$$
\int_{a}^{\sigma(b)}\left|G\left(\sigma^{2}(b), s\right)\right| \Delta s \leq \frac{1}{2}\left[\left(\sigma^{2}(b)-a\right)(\sigma(b)-a)+\frac{1}{2}(\sigma(b)-a)^{2}\right]=: K_{0,2} .
$$

As a consequence,

$$
\left\|\int_{a}^{\sigma(b)}|G(t, s)| \Delta s\right\| \leq \max \left\{K_{0,1}, K_{0,2}\right\}=: K_{0}>0
$$


If $M \neq 0,1-M^{2} \mu^{2}(t) \neq 0$ for all $t \in I, 1+e_{M}(\sigma(b), a) \neq 0$, and $1+e_{-M}(\sigma(b), a) \neq 0$, then

$$
G(t, s)=\left\{\begin{array}{cl}
\frac{1}{2 M}\left[\frac{e_{-M}(t, \sigma(s))}{1+e_{-M}(\sigma(b), a)}-\frac{e_{M}(t, \sigma(s))}{1+e_{M}(\sigma(b), a)}\right], & \text { if } a \leq \sigma(s) \leq t \leq \sigma^{2}(b), \\
\frac{1}{2 M}\left[\frac{e_{M}(t, \sigma(s)) e_{M}(\sigma(b), a)}{1+e_{M}(\sigma(b), a)}\right. & \\
\left.-\frac{e_{-M}(t, \sigma(s)) e_{-M}(\sigma(b), a)}{1+e_{-M}(\sigma(b), a)}\right], & \text { if } a \leq t<\sigma(s) \leq \sigma(b) .
\end{array}\right.
$$

We know, by [5, Theorem 2.44], that if $1+M \mu(t)>0$ and $1-M \mu(t)>0$ for all $t \in I$, then $e_{M}(t, s)>0$ and $e_{-M}(t, s)>0$ for all $(t, s) \in \mathbb{T} \times I$, so that, we only study these values of $M \in \mathbb{R}$.

From the expression of Green's function and [5, Theorem 2.39], for all $t \in[a, \sigma(b)]$ we obtain

$$
\int_{a}^{\sigma(b)}|G(t, s)| \Delta s \leq \frac{1}{2|M| M}\left[\frac{e_{M}(\sigma(b), a)-1}{1+e_{M}(\sigma(b), a)}+\frac{1-e_{-M}(\sigma(b), a)}{1+e_{-M}(\sigma(b), a)}\right]=: K_{M, 1},
$$

and, if $t=\sigma^{2}(b)>\sigma(b)$, then we have that

$$
\begin{aligned}
\int_{a}^{\sigma(b)}\left|G\left(\sigma^{2}(b), s\right)\right| \Delta s \leq \frac{1}{2|M| M}[ & \frac{e_{M}\left(\sigma^{2}(b), \sigma(b)\right)\left(e_{M}(\sigma(b), a)-1\right)}{1+e_{M}(\sigma(b), a)} \\
& \left.+\frac{e_{-M}\left(\sigma^{2}(b), \sigma(b)\right)\left(1-e_{-M}(\sigma(b), a)\right)}{1+e_{-M}(\sigma(b), a)}\right] \\
=: K_{M, 2} . &
\end{aligned}
$$

As a consequence,

$$
\left\|\int_{a}^{\sigma(b)}|G(t, s)| \Delta s\right\| \leq \max \left\{K_{M, 1}, K_{M, 2}\right\}=: K_{M}>0 .
$$

Thus, from Theorem 3.4 and Remark 3.5, we deduce the following result that assures the existence and uniqueness of solutions of the dynamic equation $\left(\bar{L}_{2}\right)$ in the sector $[\alpha, \beta]$, where $\alpha$ and $\beta$ are a pair of coupled lower and upper solutions of $\left(\bar{L}_{2}\right)$.

Corollary 5.1. Assume that $M \in \mathbb{R}$ is a given constant such that $|M|<1 / \mu(t)$ for all $t \in I$, $\alpha$ and $\beta$ are a pair of coupled lower and upper solutions of the dynamic equation $\left(\bar{L}_{2}\right)$ and the function $f: I \times \mathbb{R} \rightarrow \mathbb{R}$ satisfies hypothesis ( $\mathrm{H} f$ ). If condition (3.16) (with $\mathrm{M}^{2}$ instead of $M$ ) holds for some $K \geq 0$ such that $K<1 / K_{M}$, with $K_{0}$ and $K_{M}$ defined in (5.7) and (5.11), respectively, then the dynamic equation $\left(\bar{L}_{2}\right)$ has a unique solution in $[\alpha, \beta]$.

5.1. Particular cases. Now, we introduce some examples in differential, difference, and $q$-difference equations, to apply the previous theory.

Differential equations. Let $T>0$ and $\mathbb{T}=[0, T] \subset \mathbb{R}$. We know, by [5, Theorem 1.16], that $u:[0, T] \rightarrow \mathbb{R}$ is twice $\Delta$-differentiable at $t \in[0, T]$ if and only if $u$ is twice differentiable (in the classical sense) at $t$, and $u^{\Delta^{2}}(t)=u^{\prime \prime}(t)$. 
One can verify that the operator

$$
T_{2}[M] u(t):=-u^{\prime \prime}(t)+M^{2} u(t), \quad t \in I,
$$

is regressive on $I$ and invertible on $W_{2}$ for all $M \neq 0$. Moreover, Green's function associated to the operator $T_{2}^{-1}[M]$ in $W_{2}$ is given by the following expression.

If $M=0$, then

$$
G(t, s)= \begin{cases}\frac{1}{2}\left[\frac{T}{2}-t+s\right], & \text { if } 0 \leq s<t \leq T, \\ \frac{1}{2}\left[\frac{T}{2}-s+t\right], & \text { if } 0 \leq t \leq s \leq T .\end{cases}
$$

If $M \neq 0$, then

$$
G(t, s)= \begin{cases}\frac{1}{2 M}\left[\frac{e^{-M(t-s)}}{1+e^{-M T}}-\frac{e^{M(t-s)}}{1+e^{M T}}\right], & \text { if } 0 \leq s<t \leq T, \\ \frac{1}{2 M}\left[\frac{e^{M(T+t-s)}}{1+e^{M T}}-\frac{e^{-M(T+t-s)}}{1+e^{-M T}}\right], & \text { if } 0 \leq t \leq s \leq T .\end{cases}
$$

From the previous expressions we obtain that if $M=0$, then

$$
\left\|\int_{0}^{T}|G(t, s)| d s\right\| \leq \frac{5 T^{2}}{4}=: K_{0}>0,
$$

and if $M \neq 0$, then

$$
\left\|\int_{0}^{T}|G(t, s)| d s\right\| \leq \frac{1}{2|M| M}\left[\frac{e^{M T}-1}{1+e^{M T}}+\frac{1-e^{-M T}}{1+e^{-M T}}\right]=: K_{M}>0 .
$$

Therefore, from Corollary 5.1 we obtain the following result.

Corollary 5.2. Assume that $M \in \mathbb{R}$ is a given constant, $\alpha$ and $\beta$ are a pair of coupled lower and upper solutions of the differential equation $\left(\bar{L}_{2}\right)$, and $f: I \times \mathbb{R} \rightarrow \mathbb{R}$ is a continuous function. Assuming condition (3.16) (with $M^{2}$ instead of $M$ ) for some $K \geq 0$ such that $K<1 / K_{M}$, with $K_{0}$ and $K_{M}$ defined in (5.15) and (5.16), respectively, then the differential equation $\left(\bar{L}_{2}\right)$ has a unique solution in $[\alpha, \beta]$.

Difference equations. Let $h>0, P \in \mathbb{N} ; P \geq 2$, and $\mathbb{T}=\{0, h, \ldots, h P\} \subset \mathbb{R}$.

Given $u: \mathbb{T} \rightarrow \mathbb{R}$, it follows from [5, Theorem 1.16] that for every $t \in \mathbb{T}^{\kappa^{2}}, u$ is twice $\Delta$-differentiable at $t$, moreover, we have that

$$
u^{\Delta^{2}}(t)=\frac{u(t+2 h)-2 u(t+h)+u(h)}{h^{2}}
$$


In this case, one can verify that for all $M \in \mathbb{R}$ such that $|M| \neq 1 / h, 1+(1+M h)^{P-1} \neq 0$, and $1+(1-M h)^{P-1} \neq 0$, the operator

$$
\bar{T}_{2}[M] u(t):=\frac{-u(t+2 h)+2 u(t+h)-\left(1-M^{2} h^{2}\right) u(h)}{h^{2}}, \quad t \in I,
$$

is regressive on $I$ and invertible on $W_{2}$. Now, the function $G$ is given by the following expression.

If $M=0$, then

$$
G(t, s)= \begin{cases}\frac{1}{2}\left[\frac{h(P-1)}{2}-t+s+h\right], & \text { if } 0 \leq s+h \leq t \leq h P \\ \frac{1}{2}\left[\frac{h(P-1)}{2}-s-h+t\right], & \text { if } 0 \leq t \leq s \leq h(P-2) .\end{cases}
$$

If $M \neq 0,|M| \neq 1 / h, 1+(1+M h)^{P-1} \neq 0$, and $1+(1-M h)^{P-1} \neq 0$, then

$$
G(t, s)= \begin{cases}\frac{1}{2 M}\left[\frac{(1-M h)^{(t-s-h) / h}}{1+(1-M h)^{P-1}}-\frac{(1+M h)^{(t-s-h) / h}}{1+(1+M h)^{P-1}}\right], & \text { if } 0 \leq s+h \leq t \leq h P, \\ \frac{1}{2 M}\left[\frac{(1+M h)^{(P h+t-s-2 h) / h}}{1+(1+M h)^{P-1}}-\frac{(1-M h)^{(P h+t-s-2 h) / h}}{1+(1-M h)^{P-1}}\right], & \text { if } 0 \leq t \leq s \leq h(P-2) .\end{cases}
$$

From these expressions, we deduce the following estimates.

If $M=0$, then we have that

$$
\left\|h \cdot \sum_{s=0}^{h(P-2)}|G(t, s)|\right\| \leq \frac{5[h(P-1)]^{2}}{4}=: K_{0}>0 .
$$

If $|M|<1 / h, M \neq 0$, then we arrive at

$$
\begin{aligned}
\left\|h \cdot \sum_{s=0}^{h(P-2)}|G(t, s)|\right\| & \leq \frac{1}{2|M| M}\left[\frac{(1+M h)^{P-1}-1}{1+(1+M h)^{P-1}}+\frac{1-(1-M h)^{P-1}}{1+(1-M h)^{P-1}}\right] \\
& =: K_{M}>0 .
\end{aligned}
$$

As a consequence, we arrive at the following result.

Corollary 5.3. Assume that $M \in \mathbb{R}$ is a given constant such that $|M|<1 / h, \alpha$ and $\beta$ are a pair of coupled lower and upper solutions of the dynamic equation $\left(\bar{L}_{2}\right)$, and the function $f: I \times \mathbb{R} \rightarrow \mathbb{R}$ verifies that for every $t \in I, f(t, \cdot) \in C(\mathbb{R})$. If (3.16) (with $M^{2}$ instead of $M$ ) holds for some $K \geq 0$ such that $K<1 / K_{M}$, with $K_{0}$ and $K_{M}$ defined in (5.21) and (5.22), respectively, then the dynamic equation $\left(\bar{L}_{2}\right)$ has a unique solution in $[\alpha, \beta]$.

$q$-difference equations. Given $q \in \mathbb{R}, q>1$, and $N \in \mathbb{N}, N \geq 2$, let $\mathbb{T}=\left\{1, q, \ldots, q^{N}\right\} \subset \mathbb{R}$. 
If $u: \mathbb{T} \rightarrow \mathbb{R}$, then we know, by [5, Theorem 1.16], that for every $t \in \mathbb{T}^{\kappa^{2}}, u$ is twice $\Delta$-differentiable at $t$ and

$$
u^{\Delta^{2}}(t)=\frac{u\left(q^{2} t\right)-(q+1) u(q t)+q u(t)}{(q-1)^{2} q t^{2}} .
$$

If $t=q^{i}$ and $s=q^{j}$, then one can verify that Green's function is given by the following expression.

If $M=0$, then

$$
G(t, s)= \begin{cases}\frac{1}{2}\left[\frac{q^{N}-1}{2}-q^{i}+q^{j+1}\right], & \text { if } 0 \leq j+1 \leq i \leq N, \\ \frac{1}{2}\left[\frac{q^{N}-1}{2}-q^{j+1}+q^{i}\right], & \text { if } 0 \leq i \leq j \leq N-2 .\end{cases}
$$

If $M \neq 0,|M| \neq 1 /(q-1) q^{k}$ for each $k \in J_{N-2}=\{0,1, \ldots, N-2\}, 1+\prod_{k=0}^{N-2}(1+M(q-$ 1) $\left.q^{k}\right) \neq 0$ and $1+\prod_{k=0}^{N-2}\left(1-M(q-1) q^{k}\right) \neq 0$, then

$$
G(t, s)=\left\{\begin{array}{c}
\frac{1}{2 M}\left[\frac{\prod_{k=j+1}^{i-1}\left(1-M(q-1) q^{k}\right)}{1+\prod_{k=0}^{N-2}\left(1-M(q-1) q^{k}\right)}-\frac{\prod_{k=j+1}^{i-1}\left(1+M(q-1) q^{k}\right)}{1+\prod_{k=0}^{N-2}\left(1+M(q-1) q^{k}\right)}\right], \\
\text { if } 0 \leq j+1 \leq i \leq N, \\
\frac{1}{2 M}\left[\frac{\prod_{k \in I_{i, j}}\left(1-M(q-1) q^{k}\right)}{1+\prod_{k=0}^{N-2}\left(1-M(q-1) q^{k}\right)}-\frac{\prod_{k \in I_{i, j}}\left(1+M(q-1) q^{k}\right)}{1+\prod_{k=0}^{N-2}\left(1+M(q-1) q^{k}\right)}\right], \\
\text { if } 0 \leq i \leq j \leq N-2,
\end{array}\right.
$$

with $I_{i, j}$ defined in (4.23).

From the expression of Green's function, we obtain the following upper bounds.

If $M=0$, then

$$
(q-1) \cdot\left(\max _{0 \leq i \leq N}\left\{\sum_{j=0}^{N-2} q^{j}\left|G\left(q^{i}, q^{j}\right)\right|\right\}\right) \leq \max \left\{K_{0,1}, K_{0,2}\right\}=: K_{0}>0,
$$

with

$$
\begin{gathered}
K_{0,1}:=\frac{5\left(q^{N-1}-1\right)^{2}}{4} \\
K_{0,2}:=\frac{1}{2}\left[\left(q^{N}-1\right)\left(q^{N-1}-1\right)+\frac{1}{2}\left(q^{N-1}-1\right)^{2}\right] .
\end{gathered}
$$

If $|M|<1 /(q-1) q^{N-2}, M \neq 0$, then

$$
(q-1) \cdot\left(\max _{0 \leq i \leq N}\left\{\sum_{j=0}^{N-2} q^{j}\left|G\left(q^{i}, q^{j}\right)\right|\right\}\right) \leq \max \left\{K_{M, 1}, K_{M, 2}\right\}=: K_{M}>0
$$


where

$$
\begin{gathered}
K_{M, 1}:=\frac{1}{2|M| M}\left[\frac{\prod_{k=0}^{N-2}\left(1+M(q-1) q^{k}\right)-1}{1+\prod_{k=0}^{N-2}\left(1+M(q-1) q^{k}\right)}+\frac{1-\prod_{k=0}^{N-2}\left(1-M(q-1) q^{k}\right)}{1+\prod_{k=0}^{N-2}\left(1-M(q-1) q^{k}\right)}\right], \\
K_{M, 2}:=\frac{1}{2|M| M}\left[\frac{\prod_{k=0}^{N-1}\left(1+M(q-1) q^{k}\right)-1-M(q-1) q^{N-1}}{1+\prod_{k=0}^{N-2}\left(1+M(q-1) q^{k}\right)}\right. \\
\left.+\frac{1-M(q-1) q^{N-1}-\prod_{k=0}^{N-1}\left(1-M(q-1) q^{k}\right)}{1+\prod_{k=0}^{N-2}\left(1-M(q-1) q^{k}\right)}\right] .
\end{gathered}
$$

From the previous expressions, we have the following result.

Corollary 5.4. Assume that $M \in \mathbb{R}$ is such that $|M|<1 /(q-1) q^{N-2}, \alpha$ and $\beta$ are a pair of coupled lower and upper solutions of the dynamic equation $\left(\bar{L}_{2}\right)$, and $f: I \times \mathbb{R} \rightarrow \mathbb{R}$ verifies that for every $t \in I, f(t, \cdot) \in C(\mathbb{R})$. If (3.16) (with $M^{2}$ instead of $M$ ) is true for some $K \geq 0$ such that $K<1 / K_{M}$, with $K_{0}$ and $K_{M}$ defined in (5.26) and (5.28), respectively, then the dynamic equation $\left(\bar{L}_{2}\right)$ has a unique solution in $[\alpha, \beta]$.

\section{Acknowledgments}

The authors thank the referees of the paper for their interesting comments and suggestions. This research was partially supported by D. G. I. and F.E.D.E.R. project BFM20013884-C02-01, and by Xunta de Galicia and F.E.D.E.R. project PGIDIT020XIC20703PN, Spain.

\section{References}

[1] R. P. Agarwal, M. Bohner, D. O'Regan, and A. Peterson, Dynamic equations on time scales: a survey, J. Comput. Appl. Math. 141 (2002), no. 1-2, 1-26.

[2] R. P. Agarwal, M. Bohner, and A. Peterson, Inequalities on time scales: a survey, Math. Inequal. Appl. 4 (2001), no. 4, 535-557.

[3] R. P. Agarwal, A. Cabada, and V. Otero-Espinar, Existence and uniqueness results for $n$th order nonlinear difference equations in presence of lower and upper solutions, Arch. Inequal. Appl. 1 (2003), no. 3-4, 421-431.

[4] R. P. Agarwal, A. Cabada, V. Otero-Espinar, and S. Dontha, Existence and uniqueness of solutions for anti-periodic difference equations, to appear in Arch. Inequal. Appl.

[5] M. Bohner and A. Peterson, Dynamic Equations on Time Scales. An Introduction with Applications, Birkhäuser Boston, Massachusetts, 2001.

[6] _ Advances in Dynamic Equations on Time Scales, Birkhäuser Boston, Massachusetts, 2003.

[7] H. Brezis, Analyse Fonctionnelle: Théorie et Applications [Functional Analysis: Theory and Applications], Collection Mathématiques Appliquées pour la Maîtrise, Masson, Paris, 1983.

[8] A. Cabada, The method of lower and upper solutions for periodic and anti-periodic difference equations, to appear in Elect. Trans. Num. Anal.

[9] Extremal solutions and Green's functions of higher order periodic boundary value problems in time scales, J. Math. Anal. Appl. 290 (2004), no. 1, 35-54.

[10] A. Cabada and J. J. Nieto, Fixed points and approximate solutions for nonlinear operator equations. Fixed point theory with applications in nonlinear analysis, J. Comput. Appl. Math. 113 (2000), no. 1-2, 17-25. 


\section{Higher-order antiperiodic dynamic equations}

[11] D. Franco, J. J. Nieto, and D. O'Regan, Anti-periodic boundary value problem for nonlinear first order ordinary differential equations, Math. Inequal. Appl. 6 (2003), no. 3, 477-485.

[12] S. Hilger, Ein Maßkettenkalkül mit Anwendung auf Zentrumsmannigfaltigkeiten, Ph.D. thesis, Universität Würzburg, Würzburg, 1988.

[13] B. Kaymakçalan, V. Lakshmikantham, and S. Sivasundaram, Dynamical Systems on Measure Chains, Kluwer Academic Publishers, Dordrecht, 1996.

[14] J. Mawhin, Analyse. Fondements, Techniques, Évolution [Analysis. Foundations, Techniques, Evolution], second ed., Accès Sciences, De Boeck University, Brussels, 1997.

Alberto Cabada: Departamento de Análise Matemática, Facultade de Matemáticas, Universidade de Santiago de Compostela, 15782 Santiago de Compostela, Galicia, Spain

E-mail address: cabada@usc.es

Dolores R. Vivero: Departamento de Análise Matemática, Facultade de Matemáticas, Universidade de Santiago de Compostela, 15782 Santiago de Compostela, Galicia, Spain

E-mail address: lolirv@usc.es 